\title{
LINES COMMON TO FOUR LINEAR COMPLEXES.
}

BY DR. VIRGIL SNYDER.

(Read at the February meeting of the Society, 1897.)

In his discussion of the invariants of one or more linear complexes, Klein* makes the statement that four such complexes have two lines in common, which become coincident when the combinant of the four complexes vanishes, but otherwise the reality of the lines is not discussed.

The corresponding criterion for spherical geometry is of value in studying the cyclides and it can be proved by geometrical methods that the spheres common to four linear spherical complexes are real when the combinant is negative. On account of the direct interpretation of the simpler invariants from one geometry into the other, one might conclude by analogy that the same law holds here, which, however, is not the case.

For convenience, transform the quadratic relation

$$
P_{12} P_{34}+P_{13} P_{42}+P_{14} P_{23}=0
$$

by the transformation

$$
\begin{aligned}
& P_{12}=x_{1}+x_{2}, \quad P_{13}=x_{3}+x_{4}, \quad P_{14}=x_{5}+x_{6}, \\
& P_{84}=x_{1}-x_{2}, \quad P_{42}=x_{8}-x_{4}, \quad P_{28}=x_{5}-x_{6}, \text { into } \\
& x_{1}^{2}-x_{2}^{2}+x_{3}^{2}-x_{4}^{2}+x_{5}^{2}-x_{6}^{2}=0 .
\end{aligned}
$$

Let the four given complexes be

$$
\begin{gathered}
\psi_{i} \equiv a_{i} x_{1}+b_{i} x_{2}+c_{i} x_{3}+d_{i} x_{4}+e_{i} x_{5}+f_{i} x_{6}= \\
0[i=1,2,3,4] .
\end{gathered}
$$

The invariant of $\psi_{i}$ is

$$
A_{i i} \equiv a_{i}^{2}-b_{i}^{2}+c_{i}^{2}-d_{i}^{2}+e_{i}^{2}-f_{i}^{2}
$$

and the simultaneous invariant of $\psi_{i}, \psi_{k}$ is

$$
A_{i k} \equiv a_{i} a_{k}-b_{i} b_{k}+c_{i} c_{k}-d_{i} d_{k}+e_{i} e_{k}-f_{i} f_{k}
$$

Two complexes are in involution when their simultaneous invariant vanishes; a general complex is in involution with

"Differentialgleichungen in Liniengeometrie," Math. Annalen, vol. 5. 
a special one $\left(A_{i i}=0\right)$ when the axis of the special complex belongs to the general one.

Let

$$
A x_{1}+B x_{2}+C x_{3}+D x_{4}+E x_{5}+F x_{6}=0
$$

represent all the complexes in involution with every complex of the system

$$
l \psi_{1}+m \psi_{2}+n \psi_{3}+P \psi_{4}=0 .
$$

The axes of all the special complexes of (4) will then belong to every complex of (5) and therefore to $\psi_{1}, \psi_{2}, \psi_{3}, \psi_{1}$. The simultaneous invariant of (4) and (5) must vanish for all values of $l, m, n, P$, which gives four equations (by (3)) of the form

$$
a_{i} A-b_{i} B+c_{i} C-d_{i} D+e_{i} E-f_{i} F=0[i=1,2,3,4] \text {. }
$$

from which any four, as $A, B, C, D$ can be solved in terms of the other two, $E, F$. This gives

$$
\begin{gathered}
A=\frac{-E\left(\begin{array}{l}
a \\
e
\end{array}\right)+F\left(\begin{array}{l}
a \\
f
\end{array}\right)}{(a)} ; \quad B=\frac{E\left(\begin{array}{l}
b \\
e
\end{array}\right)-F\left(\begin{array}{l}
b \\
f
\end{array}\right)}{(b)} \\
C=\frac{-E\left(\begin{array}{l}
c \\
e
\end{array}\right)+F\left(\begin{array}{l}
c \\
f
\end{array}\right)}{(c)} ; \quad D=\frac{E\left(\begin{array}{l}
d \\
e
\end{array}\right)-F\left(\begin{array}{l}
d \\
f
\end{array}\right)}{(d)} \\
\text { where }(a)=(b)=(c)=(d)=\left|\begin{array}{llll}
a_{1} & b_{1} & c_{1} & d_{1} \\
a_{2} & b_{2} & c_{2} & d_{2} \\
a_{3} & b_{3} & c_{3} & d_{3} \\
a_{4} & b_{4} & c_{4} & d_{4}
\end{array}\right| \text { and }
\end{gathered}
$$

$\left(\begin{array}{l}a \\ e\end{array}\right)$ is what $(a)$ becomes when $a_{i}$ has been replaced by $e_{i}$ $[i=1,2,3,4]$, and similarly for the other forms.

These values for $A, B, C, D$ are now to be substituted in (4). This gives

$$
\begin{gathered}
E \psi^{\prime}+F \psi^{\prime \prime}=0, \text { where } \\
\psi^{\prime} \equiv-\left(\begin{array}{l}
a \\
e
\end{array}\right) x_{1}+\left(\begin{array}{l}
b \\
e
\end{array}\right) x_{2}-\left(\begin{array}{l}
c \\
e
\end{array}\right) x_{3}+\left(\begin{array}{l}
d \\
e
\end{array}\right) x_{4}+(a) x_{5}, \\
\psi^{\prime \prime} \equiv\left(\begin{array}{l}
a \\
f
\end{array}\right) x_{1}-\left(\begin{array}{l}
b \\
f
\end{array}\right) x_{2}+\left(\begin{array}{l}
c \\
f
\end{array}\right) x_{3}-\left(\begin{array}{l}
d \\
f
\end{array}\right) x_{4}+(a) x_{6} .
\end{gathered}
$$

There are two special complexes contained in (6), whose axes belong to (5). These axes are real, coincident or 
imaginary according as the combinant of $\psi^{\prime}, \psi^{\prime \prime}$ is negative, zero or positive, or according as

$$
\Phi \equiv\left[A \cdot^{\prime \prime \prime}\right]^{2}-A \cdot^{\prime} A^{\prime \prime}
$$

is positive, zero or negative, $\left[A^{\prime}, A^{\prime \prime}, A^{\prime \prime \prime \prime}\right.$ invariants of $\left.\psi^{\prime}, \psi^{\prime \prime}\right]$.

$$
\begin{gathered}
A^{\prime \prime \prime} \equiv-\left(\begin{array}{l}
a \\
e
\end{array}\right)\left(\begin{array}{l}
a \\
f
\end{array}\right)+\left(\begin{array}{l}
b \\
e
\end{array}\right)\left(\begin{array}{l}
b \\
f
\end{array}\right)-\left(\begin{array}{l}
c \\
e
\end{array}\right)\left(\begin{array}{l}
c \\
f
\end{array}\right)+\left(\begin{array}{l}
d \\
e
\end{array}\right)\left(\begin{array}{l}
d \\
f
\end{array}\right) \\
A^{\prime} \equiv\left(\begin{array}{l}
a \\
e
\end{array}\right)^{2}-\left(\begin{array}{l}
b \\
e
\end{array}\right)^{2}+\left(\begin{array}{l}
c \\
e
\end{array}\right)^{2}-\left(\begin{array}{l}
d \\
e
\end{array}\right)^{2}+(a)^{2} \\
A^{\prime \prime} \equiv\left(\begin{array}{l}
a \\
f
\end{array}\right)^{2}-\left(\begin{array}{l}
b \\
f
\end{array}\right)^{2}+\left(\begin{array}{l}
c \\
f
\end{array}\right)^{2}-\left(\begin{array}{l}
d \\
f
\end{array}\right)^{2}-(a)^{2}
\end{gathered}
$$

After a few simple reductions,

$$
\begin{gathered}
\Phi=\left[\left(\begin{array}{l}
a \\
e
\end{array}\right)\left(\begin{array}{l}
b \\
f
\end{array}\right)-\left(\begin{array}{l}
a \\
f
\end{array}\right)\left(\begin{array}{l}
b \\
e
\end{array}\right)\right]^{2}-\left[\left(\begin{array}{l}
a \\
e
\end{array}\right)\left(\begin{array}{l}
c \\
f
\end{array}\right)-\left(\begin{array}{l}
a \\
f
\end{array}\right)\left(\begin{array}{l}
c \\
e
\end{array}\right)\right]^{2}+ \\
+\left[\left(\begin{array}{l}
b \\
e
\end{array}\right)\left(\begin{array}{l}
c \\
f
\end{array}\right)-\left(\begin{array}{l}
b \\
f
\end{array}\right)\left(\begin{array}{l}
c \\
e
\end{array}\right)\right]^{2} \\
+\left[\left(\begin{array}{l}
a \\
e
\end{array}\right)\left(\begin{array}{l}
d \\
f
\end{array}\right)-\left(\begin{array}{l}
a \\
f
\end{array}\right)\left(\begin{array}{l}
d \\
e
\end{array}\right)\right]^{2}-\left[\left(\begin{array}{l}
b \\
e
\end{array}\right)\left(\begin{array}{l}
d \\
f
\end{array}\right)-\left(\begin{array}{l}
b \\
f
\end{array}\right)\left(\begin{array}{l}
d \\
e
\end{array}\right)\right]^{2}+ \\
+(a)^{2}\left[\left(\begin{array}{l}
a \\
e
\end{array}\right)^{2}-\left(\begin{array}{l}
a \\
f
\end{array}\right)^{2}-\left(\begin{array}{l}
d \\
f
\end{array}\right)-\left(\begin{array}{l}
b \\
f
\end{array}\right)\left(\begin{array}{l}
d \\
e
\end{array}\right)\right]^{2}+\left(\begin{array}{l}
b \\
f
\end{array}\right)^{2}+\left(\begin{array}{l}
c \\
e
\end{array}\right)^{2}-\left(\begin{array}{l}
c \\
f
\end{array}\right)^{2}-\left(\begin{array}{l}
d \\
e
\end{array}\right)^{2}+ \\
\left.\left(\begin{array}{l}
d \\
f
\end{array}\right)^{2}+(a)^{2}\right] .
\end{gathered}
$$

This value for $\Phi$ is now to be compared with the combinant of (5).

$$
\triangle \equiv\left|\begin{array}{llll}
A_{11} & A_{12} & A_{13} & A_{14} \\
A_{12} & A_{22} & A_{23} & A_{24} \\
A_{13} & A_{23} & A_{33} & A_{34} \\
A_{14} & A_{24} & A_{34} & A_{44}
\end{array}\right|
$$

$\Delta$ may be expressed as the product of the two rectangular arrys 


$$
\left\{\begin{array}{llll}
a_{1} b_{1} & c_{1} & d_{1} & e_{1} f_{1} \\
a_{2} b_{2} c_{2} d_{2} & e_{2} f_{2} \\
a_{3} b_{3} c_{3} & d_{3} & e_{3} f_{3} \\
a_{4} b_{4} c_{4} d_{4} e_{4} f_{4}
\end{array}\right\} \quad\left\{\begin{array}{llll}
a_{1}-b_{1} & c_{1}-d_{1} & e_{1}-f_{1} \\
a_{2}-b_{2} & c_{2}-d_{2} & e_{2}-f_{2} \\
a_{3}-b_{3} & c_{3}-d_{3} & e_{3}-f_{3} \\
a_{4}-b_{4} & c_{4}-d_{4} & e_{4}-f_{4}
\end{array}\right\}
$$

and is developed into

$$
\begin{gathered}
\Delta \equiv(a)^{2}-\left(\begin{array}{l}
d \\
e
\end{array}\right)^{2}+\left(\begin{array}{l}
d \\
f
\end{array}\right)^{2}+\left(\begin{array}{l}
a \\
e
\end{array}\right)^{2}-\left(\begin{array}{l}
a \\
f
\end{array}\right)^{2}+\left(\begin{array}{l}
b \\
c
\end{array}\right)^{2}+\left(\begin{array}{l}
b \\
f
\end{array}\right)^{2}+ \\
\left(\begin{array}{l}
c \\
e
\end{array}\right)^{2}-\left(\begin{array}{l}
c \\
f
\end{array}\right)^{2} \\
\left.+\left(a_{1} b_{2} e_{3} f_{4}\right)^{2}-\left(a_{1} c_{2} e_{8} f_{4}\right)^{2}+\left(a_{1} d_{2} e_{8} f_{4}\right)^{2}+b_{1} c_{2} e_{3} f_{4}\right)^{2}- \\
\left(b_{1} d_{2} e_{8} f_{4}\right)^{2}+\left(c_{1} d_{2} e_{3} f_{4}\right)^{2}
\end{gathered}
$$

'The first row is the same as the coefficient of $(a)^{2}$ in $\Phi$, and from determinants,

$$
(a)_{1}^{2}\left(c_{1} d_{2} e_{3} f_{4}\right)^{2}=\left[\left(\begin{array}{l}
a \\
e
\end{array}\right)\left(\begin{array}{l}
b \\
f
\end{array}\right)-\left(\begin{array}{l}
a \\
f
\end{array}\right)\left(\begin{array}{l}
b \\
e
\end{array}\right)\right]^{2} \text {, and similarly for }
$$

the other terms, then $\Delta \equiv(a)^{2} \Phi$. The two lines common to four linear complexes are real, coincident or imaginary according as the combinant of the complexes is positive, zero or negative. This criterion may be used to find the reality of the lines cutting four given ones, by making the principal diagonal of $\Delta$ vanish, and for $A_{i k}$, the polar of the lines $i, k$.

CoRnell UNIVERSITY, January 8, 1897.

\title{
THE CUBIO RESOLVENT OF A BINARY QUARTIC DERIVED BY INVARIANT DEFINITION AND PROCESS.
}

\author{
BY PROFESSOR H. s. WHITE.
}

(Read before the Conference at Chicago, January 1, 1897.)

In the usual discussion of a binary quartic the cubic resolvent first arises as an auxiliary in factoring the quantic, or what is the same thing, in reducing it to a determinate normal form. The coefficients of this cubic, when found, prove to be rational invariants of the original quartic. Such a fact appears as a surprise, since the invariant character of the roots of the cubic is rarely made prominent at 